\title{
Lidil
}

Revue de linguistique et de didactique des langues

\section{Un atelier d'École du spectateur avec des étudiants de FLE : de la réception d'Une Chambre en Inde à l'expression créative plurilingue}

A School of the Spectator Workshop with FSL Students: From Reception of

A Room in India to Creative Multilingual Expression

Elsa Caron

\section{(2) OpenEdition}

\section{Journals}

Electronic version

URL: http://journals.openedition.org/lidil/4856

DOI: $10.4000 /$ lidil.4856

ISSN: $1960-6052$

\section{Publisher}

UGA Éditions/Université Grenoble Alpes

Printed version

ISBN: 978-2-37747-048-8

ISSN: $1146-6480$

\section{Electronic reference}

Elsa Caron, «Un atelier d'École du spectateur avec des étudiants de FLE : de la réception d'Une Chambre en Inde à l'expression créative plurilingue », Lidil [Online], 57 | 2018, Online since 01 May 2018, connection on 01 May 2019. URL : http://journals.openedition.org/lidil/4856 ; DOI : 10.4000/lidil.4856

This text was automatically generated on 1 May 2019.

(C) Lidil 


\title{
Un atelier d'École du spectateur avec des étudiants de FLE : de la réception d'Une Chambre en Inde à l'expression créative plurilingue
}

\author{
A School of the Spectator Workshop with FSL Students: From Reception of \\ A Room in India to Creative Multilingual Expression
}

Elsa Caron

\section{Introduction}

Les étudiants que nous avons encadrés durant l'année universitaire 2016-2017 à l'université de Cergy-Pontoise (désormais UCP), suivaient une formation intensive de français langue étrangère (désormais FLE), articulant apprentissage formel de la langue et ouverture artistique et culturelle. Un des ateliers liés à cette dernière thématique proposait aux étudiants de suivre un dispositif d'École du spectateur ${ }^{1}$ (Loriol \& Lallias, 2009) autour de quatre spectacles ${ }^{2}$ parmi lesquels figurait la nouvelle création d'Ariane Mnouchkine, Une Chambre en Inde ${ }^{3}$, spectacle que nous avons choisi d'analyser dans le présent article compte tenu de sa grande richesse plurilingue et pluriculturelle (sept langues parlées sur scène, un dispositif de surtitrage en français, des comédiens de nationalités et de cultures différentes, des situations dramaturgiques très variées prenant en compte cette diversité, etc.). En quoi ce spectacle peut-il constituer un outil privilégié pour permettre aux apprenants d'expérimenter la pluralité linguistique et culturel du monde contemporain et de conscientiser leur propre répertoire plurilingue, quelles pratiques de création artistique liée à la démarche de l'École du spectateur peut-on proposer autour d'Une Chambre en Inde afin d'aborder cette pluralité ? Quel est l'intérêt en didactique du FLE d'un tel accompagnement autour du spectacle vivant et quels objectifs 
langagiers et translangagiers peut-on assigner à cette démarche dans le cadre de la didactique du plurilinguisme (Coste, 2010)?

2 Au cours de cet atelier, nous avons cherché à articuler réception esthétique au sens d'expérience vécue de l'œuvre d'art (Schaeffer, 2015), pratique théâtrale d'improvisation (Aden, 2009b), et apprentissage du FLE dans une démarche actionnelle, partant de l'idée que la pratique créative d'après-spectacle proposée par l'École du spectateur permettait de s'appuyer sur la sensibilité artistique de l'apprenant tout en visant un développement de la langue-cible. La pratique théâtrale a été intégrée depuis quelques années dans les curricula en FLE (Fonio \& Genicot, 2011; Alix, Lagorgette \& Rollinat-Levasseur, 2014 ; Rollinat-Levasseur, 2015); quant à l'improvisation, elle permettrait de développer une capacité à s'ajuster à des contextes différents et à gérer l'inconnu (Aden, 2009b). Nous tenterons d'analyser cette démarche d'École du spectateur mêlant remémoration collective de la pièce et tâche créative d'improvisation en tant que support pour une appropriation des langues, considérée comme une expérience de relation à l'altérité et de transformation de soi (Castellotti, 2017). Quelle place assigner à cette pratique de réception du spectacle vivant et de création dans la didactique du plurilinguisme, autrement dit, dans quelle mesure l'articulation entre l'expérimentation de la pluralité à travers la réception du spectacle vivant et sa réactivation créative dans l'improvisation peut-elle permettre à l'apprenant de se constituer comme un sujet plurilingue et pluriculturel?

\section{Cadre théorique}

\subsection{Des théories de la réception à la didactique du plurilinguisme}

3 Nos analyses se situent à l'intersection de plusieurs champs disciplinaires, qui prennent en compte notamment les théories de la réception du texte littéraire et du spectacle vivant. Ainsi, Umberto Eco (1985) postule une participation active du lecteur dans la construction $\mathrm{du}$ sens $\mathrm{du}$ texte, ainsi qu'une œuvre ouverte sur une pluralité d'interprétations: le texte ne dit pas tout et requiert la coopération active du lecteur. S'appuyant sur ses expériences de lectures antérieures pour donner un sens à l'œuvre, le lecteur crée en outre un "horizon d'attente» (Jauss, 1978) qui lui permettrait de construire des repères pour l'interprétation. Il s'agira alors d'analyser dans nos données de quelle manière les étudiants construisent leurs interactions à partir de leurs interprétations de la pièce, et comment l'écart entre leurs attentes et leur expérience du spectacle influe sur ces interactions. Ces théories peuvent être transposées à la réception du théâtre, le spectateur co-construit le sens et invente de nouveaux rapports entre le théâtre et le monde (Ubersfeld, 1996), et, en particulier, à la scène contemporaine, qui, par son utilisation des nouvelles technologies, constitue une interface nouvelle à analyser : « un lieu d'échange et d'interaction, de transmission et de traduction, qui met en contact et fait entrer en réaction des agents, des langages, des milieux hétérogènes " (Sermon \& Ryngaert, 2012, p. 177). Une Chambre en Inde correspond bien à cette dernière définition puisque les médias mobilisés sur scène sont très nombreux (écrans multiples, surtitrages projetés sur différents éléments du décor, projection de films, etc.), et que la diversité linguistique et culturelle est un des supports de la dramaturgie. Il s'agira alors de comprendre de quelle manière l'attention des apprenants sera mobilisée par ces multiples interfaces, au risque d'un «émiettement de la réception» (ibid., p. 178), et 
comment leurs productions langagières rendront compte de cette diversité. Comment prendre en considération la variabilité linguistique et culturelle de la pièce en cherchant à ce que cette variabilité entre en résonance avec le répertoire des apprenants ? Dans le cadre de la didactique du plurilinguisme (Coste, 2010), ainsi que des approches plurielles définies comme "toute approche mettant en œuvre des activités impliquant à la fois plusieurs variétés linguistiques et culturelles " (Candelier, 2008), nous chercherons à analyser l'impact de la réception esthétique d'Une Chambre en Inde et d'une approche de création artistique sur la compétence plurilingue et pluriculturelle des apprenants que l'on peut définir comme la compétence à communiquer d'acteurs sociaux, capables de maitriser à des degrés divers plusieurs langues ainsi que l'expérience de plusieurs cultures (Conseil de l'Europe, 2001, p. 129). L'enseignement des langues aura alors pour but de « développer un répertoire langagier dans lequel toutes les capacités linguistiques trouvent leur place» (ibid., p.11), ainsi que de favoriser la prise de conscience de la pluralité des cultures.

\subsection{Entrainer « l'intelligence divergente » par le théâtre}

4 La deuxième phase de notre dispositif, comprenant la pratique théâtrale et les improvisations, mise sur la créativité artistique pour entrainer "l'intelligence divergente » de l'apprenant (Aden, 2009a), c'est-à-dire, sa capacité à changer de point de vue. En favorisant la créativité de l'apprenant, autrement dit sa "capacité d'imaginer et de donner forme à des idées, des choses, [...] trouver des solutions inédites, originales, efficaces à des problèmes » (ibid.), les démarches artistiques « œuvrent au développement d'esprits capables de changer de référent et de perspective»(ibid.). Par ailleurs, l'influence des émotions sur la cognition a été démontrée (Puozzo-Capron \& Piccardo, 2013). L'enjeu est donc pour l'enseignant de FLE de créer des espaces d'émergence de ces émotions propices à l'apprentissage (ibid.) et de réinvestissement des connaissances dans un contexte varié. Il nous semble que le théâtre, en tant que déclencheur d'émotions et d'émergence de l'empathie, «socle de la reliance » (Aden, 2010) favorise ces conditions. Dans un paradigme de l'enaction en didactique des langues, le jeu théâtral peut être perçu comme une médiation linguistique (Aden, 2012), une perception du monde à travers nos sens et la mémoire des expériences, connaissance co-déterminée par les interactions entre les sujets entre eux et le monde (ibid.). C'est précisément la perception à travers les sens en tant que spectateur et la mémoire de cette expérience du spectacle, articulées à la pratique créative de l'improvisation que nous aimerions interroger, dans une vision holistique de l'enseignement par le théâtre, tenant compte de la place du corps et des affects dans l'apprentissage des langues et des cultures (Arnold, 2006).

\subsection{Privilégier les dimensions de la réception et de la perception}

5 Ainsi, lire ou voir une œuvre, puis décrire cette œuvre avec les autres en langue étrangère, permet de privilégier les dimensions de la réception et de la perception. La démarche se fonde ainsi sur les rencontres, les expériences et les histoires singulières des apprenants (Castellotti, 2017), incitant la rencontre entre une sensibilité et une œuvre plurielle et exigeante telle qu'Une Chambre en Inde, donnant à voir et à expérimenter l'altérité des personnages, des langues et des cultures :

Le projet est d'approcher les autres et leur monde notamment et significativement

à travers l'expérience langagière, lire (dans tous les sens du terme) les œuvres, se 
transformer/transformer les autres dans la rencontre, donc «s'altér(is)er».

(Castellotti, 2017, p. 43)

La démarche d'École du spectateur intervient alors comme un révélateur de ce qui est entré en résonance chez les apprenants pendant le spectacle.

\section{Contexte, démarche et méthodologie}

\subsection{Une démarche d'École du spectateur articulée à la didactique du plurilinguisme}

Les ateliers d'École du spectateur que nous proposons et dont l'objectif est de permettre aux jeunes de tous les âges «de partager leur expérience de spectateur par l'échange collectif, et de mettre un spectacle en résonance avec de multiples centres d'intérêts et des références partagées " (Loriol \& Lallias, 2009) ont été expérimentés dans un premier temps à l'Université Grenoble Alpes ${ }^{4}$, puis à l'Université de Cergy-Pontoise, dans le cadre d'un diplôme universitaire de FLE et de cours de FLE niveau A1 du Centre de langue et d'action culturelle de l'UCP (CILFAC) 5 . Un des ateliers proposés dans ce cadre s'articulait autour de la réception d'Une Chambre en Inde, joué le 16 mars 2017 à La Cartoucherie. Douze étudiants ont participé à l'expérience: quatre étudiants vietnamiens, quatre étudiantes chinoises, trois étudiants coréens et un étudiant turc. Les niveaux de langue $\mathrm{du}$ groupe étaient très hétérogènes puisque trois étudiants avaient un niveau B1, trois autres un niveau A2 et le reste du groupe était débutant en français. Il s'agira alors de se demander dans quelle mesure une expérience de ce type peut s'adapter à des niveaux hétérogènes et comment inclure des débutants au dispositif.

Une Chambre en Inde met en scène les aventures d'une comédienne d'une troupe française séjournant à Pondichéry qui doit monter seule un nouveau spectacle. Des apparitions liées à des rêves, à des cauchemars ou à la réalité entrent alors dans la chambre où Cornélia dort. La pièce convoque sept langues sur scène : le français, l'anglais, le tamoul, le japonais, le russe, l'arabe et l'allemand. Lorsque d'autres langues que le français sont utilisées, un procédé de surtitrage est projeté à des endroits différents de la scène (un toit de maison, un caisson de pousse-pousse, un écran au-dessus des comédiens, etc.). Le théâtre contemporain privilégie l'intermédialité, la porosité des frontières tend alors à modifier la posture du spectateur: dans ce contexte, «implication émotionnelle et distanciation intellectuelle sont plus que jamais à combiner » (Ahr, 2011). L'objectif des diverses tâches de l'atelier que nous allons décrire ci-dessous consistait à reconnaitre et à décrire le plurilinguisme de la pièce, à questionner le sens de cette pluralité, puis à conscientiser son propre répertoire plurilingue à partir des traces du spectacle, et enfin, à prendre de la distance face à ce répertoire pour élaborer une nouvelle proposition créative. Voici la progression que nous avons proposée aux apprenants à partir de ces objectifs et de la dramaturgie de la pièce ${ }^{6}$ :

1. Dans un premier temps, les apprenants sont amenés à débattre autour de leurs premières impressions du spectacle à l'entracte et à faire des hypothèses rapides sur les différents sens convoqués. Ces premières impressions filmées constituent une partie de notre recueil de données.

2. Nous avons ensuite demandé aux étudiants, une semaine plus tard, de se remémorer la pièce en groupe, lors d'une activité d'analyse chorale, qui consiste à décrire de la manière la plus objective possible un spectacle. On aboutit alors à une recréation collective de la représentation qui s'appuie sur la sensibilité, la culture et les représentations de chaque 
participant: «C'est une expérience d'appropriation de la mémoire. Après l'instantané de la représentation, avec toutes ses émotions et ses embryons d'analyse, il y a en effet un deuxième spectacle qui s'écrit [...].» (Mancel, 2010) Les apprenants peuvent utiliser la langue de leur choix pour ne pas être bloqués dans leur évocation de la pièce, des traducteurs peuvent également leur venir en aide.

3. Dans un troisième temps, les apprenants sont séparés en trois groupes et doivent proposer une improvisation à partir de contraintes et de deux tâches préliminaires liées à la dramaturgie d'Une Chambre en Inde. C'est cette dernière partie de l'atelier qui nous intéresse en particulier puisqu'il s'agit de la tâche la plus créative, même si les deux premières appellent également à la création dans la mesure où il s'agit d'une interprétation et d'une co-construction. Les apprenants devaient au préalable créer une constellation de mots dans des langues différentes à partir des traces mémorielles du spectacle qui ont émergé lors de l'analyse chorale ${ }^{7}$. Cette première tâche avait pour objectif de confronter les mémoires de la pièce et l'utilisation d'un répertoire culturel et linguistique individuel. On aboutit ainsi à une constellation évoquant Une Chambre en Inde dans 9 langues différentes (français, anglais, chinois, vietnamien, coréen, turc, arabe, russe, japonais) regroupées en quelques grands thèmes que la pièce questionne comme la guerre, la politique, l'aspect multiculturel, l'amour. Ces mots ne reflètent pas forcément ce que les étudiants ont entendu pendant la pièce, mais relèvent plutôt de la mémoire du spectacle qu'ils ont créée à partir des éléments qui les ont frappés ${ }^{8}$. Les langues qu'ils utilisent pendant cet exercice sont essentiellement leur langue d'origine et le français, mais trois autres langues émergent également : l'anglais très utilisé par les apprenants en raison de la présence de débutants, le japonais et l'arabe, entendus au cours de la pièce. Les termes relevés par les étudiants dans ces deux langues n'ont pas été prononcés dans le spectacle : ils rappellent un élément vu sur scène ( "hijab ») ainsi qu'une tentative d'interprétation d'une scène («seppuku»). La construction de l'interprétation de la pièce convoque donc les représentations des apprenants, leurs références culturelles, leur sensibilité afin d'élaborer un sens (Ubersfeld, 1996). Par groupe, les apprenants devaient ensuite choisir deux mots dans cette constellation et proposer une saynète avec la contrainte suivante: qui d'autre entre dans la chambre de Cornélia? Deux langues différentes devaient être utilisées et la saynète devait ensuite être jouée devant les autres étudiants. Cette contrainte a été choisie en prenant pour appui la dramaturgie de la pièce, elle devait par ailleurs laisser une large place à l'imaginaire des étudiants. À la suite de cet atelier, nous leur avons demandé d'écrire un compte rendu du spectacle sur une page Facebook, que nous appellerons «carnet du spectateur». Ces carnets constitueront une partie des données analysées.

\subsection{Spécificités d'une recherche-action}

La posture de chercheur que nous adoptons ici participe de ce que Pierra (2011) appelle une « recherche-action existentielle et transpersonnelle », mettant en cause la séparation du chercheur de son objet de recherche. Nous avons croisé les données recueillies sur le terrain au cours des différentes phases de notre démarche, adoptant ainsi un corpus de type ethnographique : une vidéo tournée à l'entracte le 16 mars, des vidéos de l'analyse chorale et de l'atelier d'improvisation, les carnets du spectateur sur Facebook, un entretien mené avec une des apprenantes du groupe qui avait choisi Une Chambre en Inde pour un dossier de recherche, enfin, un entretien avec Rafaelle Jolivet-Pignon ${ }^{9}$, docteure en études théâtrales, et formatrice à l'ANRAT ${ }^{10}$. Il s'agira, à travers ces données, de questionner les dimensions esthétiques et littéraires du spectacle vivant couplées à l'approche artistique de l'École du spectateur comme outil privilégié pour la mise en 
question des représentations de l'apprenant et de son identité plurilingue et pluriculturelle ${ }^{11}$.

\section{De l'expérience de la diversité à l'émergence du sujet plurilingue}

\subsection{Perception de la dimension plurilingue et pluriculturelle du monde à travers le spectacle}

9 À de nombreuses reprises dans les données recueillies, les apprenants évoquent les différentes langues présentes dans le spectacle, les reliant à des scènes précises : « En fait il y a 7 langue* dans ce théâtre. D'abord j'ai écouté le français, après c'est l'anglais. Quand les acteurs et les actrices ont dansé et ont chanté, ils parlé* le indien*. Et puis c'est le japonais. Quand Tchekhov est entré dans le scène, il a parlé le russe. Et d'autres langues, je n'ai pas compris. » (Carnet de H., étudiante chinoise) Il faut noter que ce type de reconnaissance n'a été possible qu'après le travail d'analyse chorale qui permet une remémoration collective du spectacle, comme le montre le verbatim suivant. L'enseignant-animateur demande aux apprenants de distinguer les langues utilisées dans la pièce, deux apprenantes débutantes répondent en anglais :

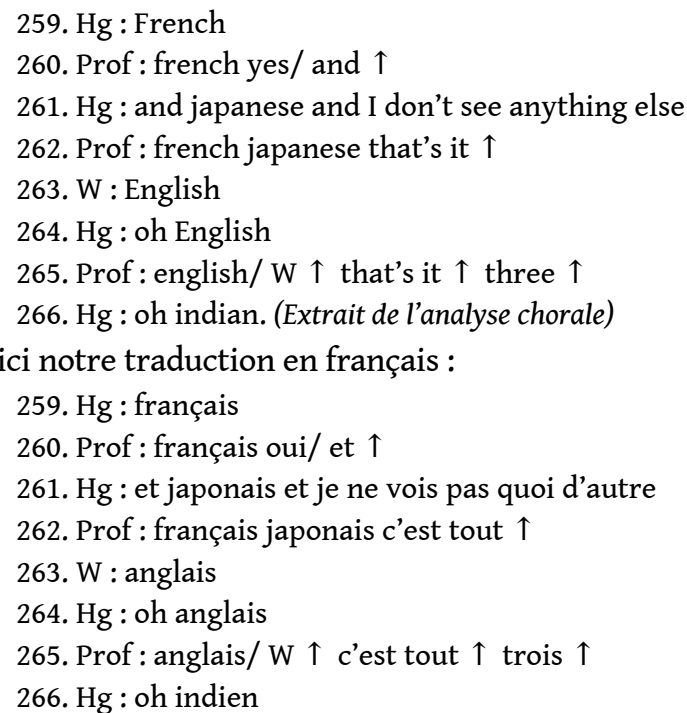

10 Ce verbatim montre que l'analyse chorale participe d'une démarche de co-construction où les apprenants sont amenés à construire ensemble la description du spectacle.

11 Les données indiquent que les apprenants se rappelaient qu'une langue de l'Inde était utilisée, mais, malgré nos précisions répétées pendant l'entracte et l'analyse chorale, le tamoul n'a pas été reconnu en tant que tel, les apprenants évoquent de manière floue «l'indien, l'hindi, le telugu (?), indian ». Cette langue est sans doute trop éloignée de leur répertoire linguistique et culturel pour qu'ils la situent précisément. La langue arabe n'a été évoquée que par l'apprenant d'origine turque, capable de situer précisément à quel moment de la pièce cette langue était utilisée, l'arabe participe de son répertoire linguistique et culturel, ce qui n'est pas le cas des autres étudiants. Ces différentes langues évoquées sont parfois reliées à des cultures: «je pense que les/ les danses et les chansons de indien $\uparrow$ c'est très/ peut-être c'est traditionnel » (vidéo de l'entracte, H., étudiante chinoise). Les tâches de réception qui convoquent la mémoire du spectateur 
permettent donc de reconstruire collectivement la dimension plurilingue du spectacle et de lui donner un sens.

Néanmoins, cette dimension plurilingue et pluriculturelle, très présente dans Une Chambre en Inde, a parfois constitué un obstacle pour la compréhension des apprenants : " cette* spectacle est très difficile pour moi + je je pas compris beaucoup de + pièces + de parties/ parce que elle parle plus vite elle parle anglais elle parle indien elle/ cette* spectacle c'est très difficile pour moi » (vidéo de l'entracte, N., étudiant vietnamien). Le surtitrage qui apparait à différents endroits du décor a cependant constitué une aide importante pour les apprenants, hormis pour les débutants pour qui ce procédé contribuait au contraire à la dispersion de l'attention. Enfin, l'aspect plurilingue de la pièce a permis une mise à distance de ses propres représentations: "The play is very multicultural and that makes me realise that French people are very curious about other countries' people. Meanwhile I know that French people are quite proud of their own language and yet they present much foreign languages in the show so it's an interesting thing ${ }^{12}$. " (Carnet de G., étudiant coréen)

En résumé, la pièce convoque la figure de «l'hyper-spectateur » : les langues participent de la nécessité de diversifier son attention en "mobilisant tour à tour sa réflexion, sa mémoire, ses sensations" (Sermon \& Ryngaert, 2012, p. 161). Cette diversification de l'attention peut être un frein à la compréhension, mais n'en reste pas moins un facteur de motivation d'apprentissage de la langue, en particulier pour les débutants: «[...] lastly, I seriously would like to break the langage barrier as soon as possible ${ }^{13}$." (Carnet de Ha., étudiante coréenne)

\subsection{Conscientiser et valoriser son propre répertoire ainsi que celui des autres en partant des traces mémorielles du spectacle}

Les apprenants ont utilisé les traces mémorielles collectives de la pièce qui ont émergé lors de l'analyse chorale pour valoriser leur propre répertoire personnel : la constellation d'une soixantaine de mots dans neuf langues différentes, le travail de lecture à voix haute, permet de valoriser ses répertoires et de découvrir ceux des autres. Par ailleurs, les données montrent que certains apprenants ont demandé à des traducteurs dont la langue première était différente de la leur de les aider à écrire et à lire des mots dans une langue qu'ils ne connaissaient pas (le russe par exemple, qui faisait également partie des langues de la pièce). Il s'agit donc d'un travail collectif et actif sur la valorisation des répertoires des participants de l'atelier, aboutissant au développement de compétences plurilingues et pluriculturelles :

Toutes les langues et variétés présentes dans l'école [...] sont considérées comme des « langues de l'éducation » en ce que toutes contribuent ou sont susceptibles de contribuer, que les élèves les possèdent déjà ou les apprennent ou y soient simplement exposés, à leur expérience scolaire de la pluralité linguistique, au développement de leurs capacités plurilingues et à la construction de leurs connaissances et expériences d'apprentissage. (Coste, 2010)

Les traces du spectacle et des différentes langues et cultures sur scène entrent souvent en résonance avec l'expérience de vie des apprenants, et sont donc sans doute liée à la mémoire épisodique: la mémoire des souvenirs personnels, souvent vécus dans un contexte affectif et émotionnel particulier. Une des apprenantes chinoises indique qu'elle a trouvé certains passages du spectacle "très émouvants " comme la saynète dans laquelle une femme indienne est forcée par son père à se marier : « tout le monde avoir 
avoir heu le droit pour choisir heu de se marier avec quelqu'un" (entretien de Z., étudiante chinoise). L'étudiante compare cette représentation du mariage en Inde au passé de la Chine : «avant c'est comme heu l'Inde oui c'est pareil ». Les traces de la pièce qui émergent dans les données semblent par conséquent entrer dans la construction d'une composante identitaire et d'un processus de distanciation chez l'apprenant, à travers les émotions liées au spectacle.

\subsection{Créer pour entrer en relation et s'approprier les langues et les cultures}

Une autre dimension clé apparait dans les données, celle de l'empathie, ou la capacité à se mettre à la place de l'autre tout en restant conscient d'être soi-même, et qui constitue " une compétence dans le développement de l'intercompréhension langagière et de la relation interculturelle» (Aden, 2010). Le verbatim suivant montre comment les apprenants, lors de l'analyse chorale, sont en mesure de développer la compétence d'empathie à travers la description du spectacle :

109. $\mathrm{H}$ : à la fin de la/ première partie + les gens qui jouaient la guitare (Imite le comédien qui joue de la guitare dans la pièce.)

110. $\mathrm{K}$ : avec les balais (sourire) (Imite les gestes du comédien.)

111. $\mathrm{H}$ : ah oui (rires)

À travers un jeu de miroir spontané où un des apprenants imite les gestes de son camarade, qui, lui-même, cherche à imiter les gestes des comédiens qu'il a vus sur scène, le mimétisme impliqué ici peut engager un mécanisme d'empathie et de relation à l'autre à travers la résonance motrice. Nous pouvons ici constater que l'utilisation spontanée de l'imitation ne traduit pas de lacune lexicale, mais plutôt un renforcement du souvenir évoqué. L'analyse chorale peut ainsi constituer une situation pédagogique favorable à la mise en relation des imaginaires à travers la mémoire du spectacle, ainsi qu'à la construction de la compétence d'empathie à travers l'imitation de ce qui a été vu sur scène. Il s'agit à présent de comprendre comment les apprenants se sont approprié la pluralité linguistique et culturelle du spectacle à travers l'improvisation qui devient, dans notre pratique d'École du spectateur, une nouvelle proposition créative à partir d'Une Chambre en Inde.

L'atelier d'improvisation devient lieu d'expérimentation et de création artistique plurilingue à partir des traces révélées dans les tâches précédentes, et qui entrent en résonance avec l'imaginaire des apprenants. La langue n'est alors plus au service de la communication ni de la réception, comme c'était le cas dans l'analyse chorale, mais se trouve au fondement même du jeu théâtral (Armand, Lory \& Rousseau, 2013). Voici la transcription de la proposition d'un groupe de trois apprenants (A, parlant le chinois et l'anglais, niveau A2 en français ; B, parlant le chinois et l'anglais, débutant en français ; et $S$, parlant le turc et l'anglais, débutant en français) qui ont choisi de travailler à partir des mots « Shakespeare » et « Love Story » de la constellation.

A : Oh! Comment je fais! Je suis tombée amoureuse de Shakespeare $\uparrow$ (Se tourne vers S.) Tu me manques!

$\mathrm{S}:$ To be or not to be, that's the question...

A : OH Shakespeare! (Se met à genoux et lui tend les mains.) Je t'aime! do you remember me?

$\mathrm{S}:$ (Se recule et ouvre les bras.) Tanımıyorum seni be kadın, nereden çıktın karsıma?

A : Tu dis quoi ? Je ne comprends pas... 
B : (Arrive en courant.) HE IS MINE! My Shakespeare! I know why you refused her because you love me! Let's go. (Elle prend $S$ par le bras et ils commencent à partir.)

$\mathrm{A}$ : (Va vers B et lui touche le bras.) 等下, 你是谁阿你

B : (Elle repousse A.) 干嘛, 跟你有什么关系

$\mathrm{A}:$ 我先来的

$\mathrm{B}$ : 什么, 这跟先来后到有什么关系

A : (Les poings sur les hanches.) 当然了你多少岁了, 你看着那么老

B : I'm eighteen.

A : Eighteen?

B : Yeah, I'm younger!

A : (L'air dépité.) C'est incroyable ! (S'en va déprimée.)

$\mathrm{B}$ : (Retourne vers $S$ et lui prend le bras de nouveau.) Let's go Shakespeare my love.

$\mathrm{S}$ : No I don't want you! (Se dégage et se recule.) Theater is the only thing I want for me!

B : No you don't want to tell them the truth! Tell them the truth (montre les spectateurs) you love me!

$S$ : No no I don't want you!

$\mathrm{B}:$ Are you serious?

$\mathrm{S}:$ Yeah.

B : Ok. (Elle s'en va en pleurant)

A : (L'arrête.) 你等等 你被拒绝啦, 走我们一起!

B : 走 ! 8 (Elles s'en vont bras-dessus, bras-dessous.)

$\mathrm{S}$ : (Resté seul sur scène.) Et moi $^{14}$ ? (Transcription à partir de la vidéo)

Les apprenants se sont approprié l'aspect plurilingue et pluriculturel de la pièce d'Ariane Mnouchkine, ainsi que les thématiques présentes dans Une Chambre en Inde comme le personnage de Shakespeare, l'amour et le rôle du théâtre, pour créer une nouvelle proposition, ce détour leur permettant de développer des compétences langagières et translangagières. C'est une réponse nouvelle combinant savoirs, expériences et imaginaire (Aden, 2009a) que les apprenants construisent pour répondre aux interrogations contenues dans la pièce. Dans cette saynète, la place majoritaire accordée à l'anglais est due à la présence de deux étudiants sur trois débutants en français, les apprenants ont cependant cherché à convoquer l'ensemble de leur répertoire linguistique, comme demandé dans la contrainte. L'utilisation des langues convoque une pluralité de rôles et de représentations à la fois culturelles et linguistiques que les apprenants ont su valoriser: la référence à une culture commune partagée (Galisson, 1991) «to be or not to be ", les deux langues principales le français, langue-cible, et l'anglais, langue de communication, se chevauchent sans problème de compréhension, seul le turc est conscientisé comme une langue éloignée du répertoire de la plupart des apprenants puisqu'il s'agit de la seule langue qui pose un problème de compréhension «A : je ne comprends pas », la langue chinoise, langue commune à deux apprenantes, est utilisée lorsque les deux personnages veulent se parler en aparté, mais le public n'a pas besoin de traduction pour comprendre de quoi il s'agit puisque le ton et les gestes des personnages (cf. transcription de la vidéo) suffisent pour comprendre qu'ils se disputent ; enfin, lorsque B s'adresse au public pour l'appeler à adhérer à ses propos, elle le fait dans une langue qu'elle sait connue de tous: l'anglais. La tâche proposée ici implique la capacité à utiliser un répertoire linguistique composite et à procéder à des choix reposant sur des variations interlinguistiques ainsi que sur des éléments paraverbaux: la dimension polyphonique du message est créée en fonction des ressources de l'apprenant et des représentations qu'il se fait des ressources de l'interlocuteur et du public. Les fonctions discursives sont multiples: s'adresser à une communauté linguistique et culturelle, sélectionner un ou plusieurs participants ou au contraire l'exclure, etc. Invité à 
agir en tant qu'acteur social plurilingue et à prendre en charge différentes fonctions de son discours lors de stratégies de communication qu'il met en place, l'apprenant prend conscience de son répertoire linguistique et de la diversité culturelle en relation avec l'altérité que constituent à la fois son expérience du spectacle, la relation à son interlocuteur dans l'improvisation, ainsi que la relation au public. Les apprenants font ainsi l'expérience vivante de la relation en langues étrangères à travers le spectacle "pour moi, les façons de jouer des acteurs sont intéressants, il me rend heureux" (carnet du spectateur de Nh., étudiant vietnamien) ainsi qu'à travers le jeu théâtral ils mettent en œuvre l'acte dynamique de "translangager» défini par Aden (2014, p. 115) comme un « acte dynamique de reliance à soi, aux autres et à l'environnement par lequel émergent en permanence des sens partagés entre les humains ». Cette expérience de la relation met donc en résonance l'imaginaire du spectacle Une Chambre en Inde et l'imaginaire des apprenants pour une nouvelle proposition créative engageant l'identité de l'apprenant, son corps, ses références, ses représentations. Jolivet-Pignon parle de l'importance de l'engagement personnel dans les ateliers d'improvisation d'École du spectateur: "Ce qu'on n'a pas dit c'est la manière dont ça nous engage et moi [...] je travaille sur la responsabilité, ça nous met en danger [...] la disponibilité, d'arriver d'être maximum dégagé de tous présupposés, préjugés.» (Extrait de l'entretien) La rencontre avec une pièce est alors considérée comme " une mise en danger ", le spectateur prend le risque d'être déplacé, dérangé par rapport aux codes qu'il a acquis. Il s'agit par conséquent non seulement de "libérer un mode d'expression", mais également de rendre compte de l'objet en mettant en avant sa responsabilité en tant que sujet, « lié à la sensibilité, à la vision à la formation, tout ce qui constitue celui qui va créer l'objet ».

$\mathrm{Au}$ cours de ce travail, les apprenants se sont approprié des rôles pour répondre à la contrainte, jouant même des personnages connus de tous (Barack Obama par exemple), qui les ont valorisés en tant que sujets plurilingues amenés à interagir en utilisant leur propre langue, la langue de communication (l'anglais) et la langue-cible (le français). Par ailleurs, jouer un personnage a permis à certains de se confronter à leurs difficultés linguistiques et à les transcender : nous avons été très frappée par le jeu d'un apprenant dont les difficultés en anglais et en français depuis le début de l'année étaient avérées et qui était souvent en retrait dans les cours de langue, mais qui, grâce au théâtre, a montré une véritable capacité de prise de risque en jouant le rôle principal pendant les improvisations. Propulsé au-devant de la scène, l'apprenant a utilisé trois langues (l'anglais, le français et le vietnamien) avec une aisance et une spontanéité jamais montrée jusqu'ici: la pratique théâtrale offre des situations qui permettent aux apprenants de "sortir d'eux-mêmes et de découvrir qu'ils peuvent parler français autrement qu'ils ne l'ont fait jusqu'à cette expérience » (Rollinat-Levasseur, 2015, p. 255). Ainsi, la pratique créative de la scène apparait comme une possibilité pour l'apprenant de se constituer en tant que sujet en langue étrangère, "de continuer de dire "je" en élaborant sa (ses) propre(s) parole(s) en langue étrangère » (Pierra, 2006, p. 247).

\section{Conclusion et perspectives}

21 La démarche d'École du spectateur que nous proposons ici pourrait donc être considérée comme un espace complexe d'exposition à l'altérité, de mise en relation des imaginaires et des langues et de co-création dramaturgique en langue étrangère. L'apprenant est amené à valoriser son répertoire plurilingue et celui des autres, et à entrer dans une 
démarche d'invention d'une « mémoire commune de nos expériences du monde » (Aden, 2017) à travers la description collective et collaborative d'une pièce de théâtre d'une part, et à travers l'improvisation permettant de naviguer entre les langues, développant la créativité et l'autonomie (ibid.) d'autre part. Si les apprenants ont pu vivre ensemble l'expérience de la représentation d'Une Chambre en Inde et se confronter à son altérité, un parcours complet d'École du spectateur proposant plusieurs spectacles, et prenant en compte notamment de manière plus approfondie les pratiques théâtrales intégrant une réflexion sur les langues et les cultures, et le développement de l'empathie (Aden, 2010), nous semble nécessaire pour un développement plus complet de la compétence plurilingue et pluriculturelle, en tant que maitrise de plusieurs langues et expériences des cultures, en particulier pour les apprenants débutants. Nous proposons de notre côté de réfléchir à une compétence transversale couplant capacité de médiation et d'appropriation créative des langues sur scène.

Une des difficultés reste néanmoins la rareté de la programmation de spectacles mêlant les langues et les cultures, cependant, de nombreux artistes de la scène contemporaine, notamment la troupe du Thêâtre du Soleil, sont engagés actuellement dans ce choix (Abdelkader, Bazile \& Fertat, 2014), et le théâtre contemporain peut apparaitre comme un outil majeur de transmission plurilingue et interculturelle, en tant que lieu privilégié de rencontre des langues et des cultures et de questionnements sur notre monde contemporain ${ }^{15}$. À condition de cibler des objectifs et d'adapter le protocole au publiccible, de très nombreuses pistes de travail sont possibles pour faire émerger les fantômes de Cornélia.

\section{BIBLIOGRAPHY}

AbdelKader, Yamna, BAzIle, Sandrine \& Fertat, Omar (dir). (2014). Pour un Théâtre-Monde. Plurilinguisme, interculturalité et transmission. Pessac : Presses universitaires de Bordeaux. ADEN, Joëlle. (2009a). La créativité artistique à l'école : refonder l'acte d'apprendre. La créativité dans tous ses états : enjeux et potentialités en éducation, Synergies Europe, 4, 173-180.

ADEN, Joëlle. (2009b). Improvisation dans le jeu théâtral et acquisition de stratégies d'interaction. Dans J. Aden (dir.), Apprentissage des langues et pratiques artistiques (p. 67-101). Paris : Manuscrit recherche-université.

ADEN, Joëlle. (2010). L'empathie, socle de la reliance en didactique des langues. Dans J. Aden, T. Grimshaw \& H. Penz (dir), Enseigner les langues-cultures à l'ère de la complexité: approches interdisciplinaires pour un monde en reliance / Teaching Language and Culture in an Era of Complexity: Interdisciplinarity Approaches for an Interrelated World (p. 23-44). Bruxelles : Peter Lang.

ADEN, Joëlle. (2012). La médiation linguistique au fondement du sens partagé : vers un paradigme de l'enaction en didactique des langues. Ela, 3, 267-284.

ADEN, Joëlle. (2014). Apprendre les langues par corps. Dans Y. Abdelkader, S. Bazile \& O. Fertat (dir.), Pour un Théâtre-Monde. Plurilinguisme, interculturalité et transmission. Pessac : Presses universitaires de Bordeaux. 
ADEN, Joëlle. (2017). Langues et langage dans un paradigme enactif. Recherches en didactique des langues et des cultures - Les Cahiers de l'Acedle, 14(1). Disponible en ligne sur <http:// rdlc.revues.org/1085> (consulté le 10 juin 2017).

AHR, Sylviane. (2011). Théâtre et langue(s) : interactions dans les créations contemporaines, perspectives pour la classe et la formation. Synergies France, 7, 5-10.

Alix, Christophe, Lagorgette, Dominique \& Rollinat-LeVASSeur, Ève-Marie (dir.). (2014). Didactique du français langue étrangère par la pratique théâtrale. Chambéry : Université de Savoie. ARmAnd, Françoise, LoRy, Marie-Paule \& Rousseau, Cécile. (2013). « Les histoires, ça montre les personnes dedans, les feelings. Pas possible si pas de théâtre. » (Tahina) - Ateliers d'expression théâtrale plurilingues en classe d'accueil. Lidil, 48, 37-55. Disponible en ligne sur <http:// journals.openedition.org/lidil/3311>.

ARNOLD, Jane. (2006). Comment les facteurs affectifs influencent-ils l'apprentissage d'une langue étrangère ? Ela, 4, 407-425.

CANDELIER, Michel. (2008). Approches plurielles, didactiques du plurilinguisme : le même et l'autre. Les Cahiers de l'Acedle, 5(1), 65-90.

CONSEIL DE L'EUROPE. (2001). Cadre européen commun de référence pour les langues : apprendre, enseigner, évaluer. Paris : Didier.

Coste, Daniel. (2010). Diversité des plurilinguismes et formes de l'éducation plurilingue et interculturelle. Les Cahiers de l'Acedle, Notions en questions en didactique des langues - Les plurilinguismes, 7(1), 141-165.

CAstellotti, Véronique. (2017). Pour une didactique de l'appropriation. Paris : Didier.

DANAN, Joseph. (2010). Qu'est-ce que la dramaturgie? Paris : Actes Sud.

Eco, Umberto. (1985). Lector in fabula. Paris : Grasset.

FonIO, Filippo \& GENICOT, Geneviève. (2011). The Compatibility of Drama Language Teaching and CEFR Objectives-Observations on a Rationale for an Artistic Approach to Foreign Language Teaching at an Academic Level. Scenario. Journal for Drama and Theater in Foreign and Second Langauge Education, 5(2), 75-89.

GALISSON, Robert. (1991). De la langue à la culture par les mots. Paris : CLE International.

JAUSS, Hans-Robert. (1978). Pour une esthétique de la réception. Paris : Gallimard.

LORIOL, Jean-Pierre \& LALLIAS, Jean-Claude. (2009). L'École du spectateur, c'est quoi au juste ? Trait d'Union : lettre aux adhérents de l'Anrat, 17.

MANCEL, Yannic. (2010). Entretien avec Claire Rannou. Dans Continuum, 1, \#la transmission, ANRAT, 6-13.

PIERRA, Gisèle. (2006). Le corps, la voix, le texte. Arts du langage en langue étrangère. Paris :

L'Harmattan.

PIERRA, Gisèle. (2011). Pratique théâtrale en FLE : spécificités d'une recherche action en didactique. Synergies Chine, 6, 105-114.

PuOZZo-CAPRON, Isabelle \& PICCARDO, Enrica. (2013). Au commencement était l'émotion : Introduction. Lidil, 48, 5-16.

ROLLINAT-LEVASSEUR, Ève-Marie. (2015). La littérature en acte : voir, entendre, ressentir. Dans A. Godard, La littérature dans l'enseignement du FLE (p. 222-264). Paris : Didier. 
SCHAEFFER, Jean-Marie. (2015). L'expérience esthétique. Paris : Gallimard.

SERMON, Julie, \& RYNGAERT, Jean-Pierre. (2012). Théâtres du XXI siècle : commencements. Paris :

Armand Colin.

UBERSFELD, Anne. (1996). Lire le théâtre II. L'école du spectateur. Paris : Belin.

\section{Autres documents}

Charte nationale de l'École du spectateur. (2010). Disponible en ligne sur <www.anrat.net/pages/ ecole-du-spectateur> (consulté le 10 juin 2017).

\section{NOTES}

1. L'École du spectateur est une pratique d'éducation artistique et culturelle d'accompagnement au spectacle vivant, décrite entre autres dans la Charte nationale de l'École du spectacle, disponible en ligne sur : <www.anrat.net/pages/ecole-du-spectateur>.

2. L'analyse de l'ensemble du dispositif fait actuellement l'objet de notre thèse de doctorat.

3. L'auteur tient à remercier l'équipe du Théâtre du Soleil pour son accueil et sa disponibilité, ainsi que pour le bon déroulement de l'atelier.

4. L'UGA a proposé en 2015-2016 ainsi qu'en 2016-2017 deux projets autour de l'interculturel et de la réception du spectacle vivant. Ces projets ont été financés par le programme Promising et ont permis à quarante jeunes français et internationaux de voir trois spectacles à la MC2 de Grenoble et de proposer un travail collaboratif et plurilingue autour de ces sorties.

5. Le CILFAC est devenu Centre de langue française (CLF) depuis le $1^{\mathrm{er}}$ janvier 2018 à l'Université de Cergy-Pontoise.

6. Joseph Danan postule une définition réunissant les deux sens du mot dramaturgie (art de la composition des pièces et pensée du passage à la scène des pièces de théâtre) et qu'il définit comme « organisation de l'action » (2010, p. 7-8). Actualisée sur scène par les choix de la mise en scène, elle est ouverte au travail du spectateur et à son « appropriation » (ibid., p. 40).

7. La démarche de l'École du spectateur mise en œuvre dans le cadre de cet atelier fournit de très nombreuses pistes d'activités possibles à partir des pièces vues : l'atelier proposé ici aurait pu préparer davantage les étudiants à l'action dramatique en leur demandant, sous la forme du théâtre-image par exemple, d'explorer des postures de Cornélia ou de ses fantômes, ou encore, à travers des micro-actions, de rejouer des situations ou des émotions.

8. Il faudra alors interroger les étudiants en entretien semi-dirigé pour comprendre pourquoi ils se souviennent de tels éléments et pas d'autres, pourquoi ces éléments entrent en résonance avec leurs représentations : ce travail fait partie actuellement de notre thèse de doctorat.

9. Rafaelle Jolivet-Pignon est l'auteure de La Représentation rhapsodique. Quand la scène invente le texte, Paris : L'Entretemps, 2015.

10. L'ANRAT (Association nationale de recherche et d'action théâtrale) propose de nombreuses formations autour de l'analyse chorale et de l'École du spectateur à destination des enseignants, mais également des éducateurs, des animateurs et des professionnels du théâtre.

11. Un travail approfondi autour des séquences potentiellement acquisitionnelles (SPA) devra également être mené autour des données collectées afin d'analyser le développement des compétences langagières des apprenants.

12. Notre traduction: «La pièce est très multiculturelle et cela me fait penser que les Français sont très curieux des gens venant d'autres pays. En même temps, je sais que les Français sont plutôt fiers de leur propre langue et pourtant ils présentent plusieurs langues étrangères dans le spectacle, donc c'est intéressant. » 
13. Notre traduction : " [...] enfin, j'aimerais vraiment briser la barrière de la langue aussi vite que possible. »

14. A : Oh! Comment je fais! Je suis tombée amoureuse de Shakespeare! Tu me manques!

B : Être ou ne pas être, telle est la question...

A : Oh Shakespeare ! Je t'aime! Est-ce que tu te souviens de moi?

$\mathrm{S}:$ Je ne te connais pas, femme, d'où viens-tu?

A : Tu dis quoi ? Je ne comprends pas...

B : Il est à moi ! Mon Shakespeare! Je sais pourquoi tu l'as repoussée : parce que tu m'aimes ! Viens.

A : Attends, tu es qui toi?

B : Quoi ? De quoi te mêles-tu?

A : Je l'ai rencontré la première!

B : Quoi ? Pour l'amour, il n'y a pas de tôt ou de tard.

A : Bien sûr que si! Quel âge as-tu? Tu as l'air bien vieille.

$B$ : J'ai dix-huit ans.

A : dix-huit?

B : oui je suis plus jeune!

A : C'est incroyable!

B : Partons Shakespeare mon amour.

$\mathrm{S}$ : Non, je ne te veux pas! Le théâtre est la seule chose qui m'importe!

$B$ : Non tu ne veux pas leur dire la vérité ! Dis-leur la vérité, tu m'aimes !

$\mathrm{S}:$ Non non je ne veux pas de toi!

B : Tu es sérieux?

A : Oui.

B : Ok.

A : Attends, il t'a repoussé aussi ? Oh là là partons.

B : Partons!

$\mathrm{S}:$ Et moi?

15. Le réseau Canopé proposera en janvier 2018 une plateforme numérique (Théâtre en Acte) dédiée à la comparaison d'extraits de mises en scène différentes d'une même pièce accompagnés d'un parcours pédagogique inspiré de l'École du spectateur.

\section{ABSTRACTS}

Our contribution tries to analyze a "School of the Audience" approach applied to the Ariane Mnouchkine's piece A Room In India, connecting aesthetic reception, practice of theatre linked to this reception, and didactics of multilingualism. How does this kind of approach allow learners to discover the linguistic and cultural plurality of their environment, whilst becoming aware of their own repertoire? To what extend does an approach based on the perceptions, the emotions and the creativity of the learner go along with a process of language appropriation? Bringing together reception theories, theatre in French as a foreign language along with didactics of multilingualism and appropriation, our contribution describes and analyzes the link between perception of the multilingual and multicultural dimension of the world and the building of the multilingual subject through that approach. 
Notre contribution cherche à analyser une démarche d'École du spectateur mise en œuvre autour du spectacle Une Chambre en Inde d'Ariane Mnouchkine, articulant réception esthétique, pratique théâtrale liée à cette réception, et didactique du plurilinguisme. En quoi une démarche de ce type peut-elle permettre aux apprenants de découvrir la pluralité linguistique et culturelle de leur environnement tout en conscientisant leur propre répertoire ? Dans quelle mesure un dispositif qui s'appuie sur les perceptions, les émotions et la créativité de l'apprenant peut-il accompagner un processus d'appropriation des langues? Confrontant les théories de la réception, du théâtre en français langue étrangère, ainsi que la didactique du plurilinguisme et de l'appropriation, notre contribution propose de décrire et d'analyser l'articulation entre perception de la dimension plurilingue et pluriculturelle du monde et construction du sujet plurilingue à travers cette démarche.

\section{INDEX}

Keywords: French as a foreign language, theatre, "School of the Audience", aesthetic reception, artistic approaches, didactics of multilingualism, appropriation

Mots-clés: français langue étrangère, théâtre, École du spectateur, réception esthétique, démarches créatives, didactique du plurilinguisme, appropriation

\section{AUTHOR}

\section{ELSA CARON}

Université de Cergy-Pontoise \& ENS Lyon, ICAR, ED3LA 